\title{
Serum response factor induces epithelial to mesenchymal transition with resistance to sorafenib in hepatocellular carcinoma
}

\author{
JUN SANG BAE ${ }^{1}$, SANG JAE NOH $^{1}$, KYOUNG MIN KIM $^{1}$, KYU YUN JANG ${ }^{1}$, \\ MYOUNG JA CHUNG ${ }^{1}$, DAE GOHN KIM ${ }^{2}$ and WOO SUNG MOON ${ }^{1}$
}

\author{
Departments of ${ }^{1}$ Pathology and ${ }^{2}$ Internal Medicine, Chonbuk National University, Medical School, \\ Research Institute of Clinical Medicine of Chonbuk National University Hospital and \\ Research Institute for Endocrine Sciences, Jeonju 561-756, Republic of Korea
}

Received August 6, 2013; Accepted September 25, 2013

DOI: 10.3892/ijo.2013.2154

\begin{abstract}
The epithelial to mesenchymal transition (EMT) is a crucial process in tumor progression. EMT of tumor cells not only causes increased metastasis, but also contributes to drug resistance. Serum response factor (SRF) is a transcription factor that plays a central role in carcinogenesis and tumor progression in several types of cancers. We investigated the effect of EMT-related SRF, focusing on its promotion of chemoresistance against sorafenib in hepatocellular carcinoma (HCC). We examined SRF and Snail expression in 146 cases of HCCs by immunohistochemistry. We also examined the chemoresistance effect of SRF in HCC cells by transfecting HLE cells with SRF cDNA and SH-J1 cells with SRF antisense cDNA. Expression of SRF and Snail were detected in $37.6 \%$ (55 of 146 cases) and in 12.3\% (18 of 146 cases) of the HCCs, respectively. None of the tumor-free liver tissues showed SRF or Snail expression. SRF expression was closely correlated with the expression of Snail $(\mathrm{p}<0.001)$ and expression of both SRF and Snail showed significant correlation with the high histological grade ( $\mathrm{p}=0.015$ and 0.003 , respectively). Overexpression of SRF in HLE cells led to increased expression of mesenchymal markers, as well as increased cell growth and colony formation. Overexpression of SRF also led to a significant reduction in the cytotoxic effect of sorafenib in HLE cells. Conversely, inhibition of SRF expression in the SH-J1 cells significantly enhanced the apoptotic effects of sorafenib, along with the reduced expression of mesenchymal markers and restored the expression of E-cadherin. These results suggest that SRF is critical for $\mathrm{HCC}$ to acquire a
\end{abstract}

Correspondence to: Professor Woo Sung Moon, Department of Pathology, Chonbuk National University, Medical School, 567 Baekjedaero, Deokjin-gu, Jeonju-si, Jeollabuk-do 561-756, Republic of Korea E-mail:mws@chonbuk.ac.kr

Key words: carcinoma, hepatocellular, serum response factor, chemoresistance mesenchymal phenotype, which leads to resistance against a sorafenib-mediated apoptotic effect.

\section{Introduction}

Hepatocellular carcinoma (HCC) is the fifth most common malignant tumor in men, and the seventh in women, making it the third-leading cause of cancer-related death in the world $(1,2)$. Although significant progress has been made in screening for early detection, the overall prognosis for patients with advanced $\mathrm{HCC}$ remains poor (3). The mortality of patients with $\mathrm{HCC}$ is predominantly the result of tumor invasion and metastasis, and epithelial-mesenchymal transition (EMT) is a crucial biological process in cancer progression, invasion and metastasis (4). EMT is typically characterized by the impaired expression or function of E-cadherin, a protein that promotes cell-to-cell contact and activation of transcription programs that promote a fibroblast-like, invasive phenotype (5). Moreover, the development of an EMT phenotype in cancer cells may also result in enhanced resistance to chemotherapy and radiation therapy, further contributing to the neoplastic phenotype (6-9). The zinc-finger factor, Snail, is one of several transcription factors that strongly repress $\mathrm{CDH}$ ( the gene that encodes E-cadherin) and is thought to be an inducer of EMT (10).

Serum response factor (SRF) is a transcription factor of the MADS-box family and it is involved in the regulation of many genes including immediate early genes, like $c$-Fos, Jun and $E g r$, as well as tissue-specific genes involved in cellular activities such as proliferation, migration, differentiation, angiogenesis and apoptosis (11-15). Accumulating evidence has suggested that SRF plays multiple roles in carcinogenesis and tumor progression in various cancers, specifically in the mesenchymal transition of epithelial tumor cells (16-20). Sorafenib is an oral multi-kinase inhibitor approved by the US Food and Drug Administration for the treatment of patients with unresectable HCC $(21,22)$. The significant survival benefit in sorafenib-treated patients with unresectable $\mathrm{HCC}$ has been reported, although drug resistance restricts the therapeutic efficacy of the treatment $(3,23)$. Therefore, it is important to understand the various mechanisms contributing to acquired drug resistance against sorafenib; EMT may also play an important role in development of drug resistance. While 
SRF has been documented as an important function in EMT of cancer, the role of SRF in the resistance of HCC cells to sorafenib has not been elucidated.

In the present study, we examined: i) the expression of SRF in surgical specimens of HCC and the relationship between SRF expression and clinicopathologic factors; ii) the relationship between expression of SRF and Snail, a suppressive transcriptional factor of E-cadherin in HCC tissues; iii) the phenotypic change induced in $\mathrm{HCC}$ cells after exogenous modulation of SRF expression; and iv) the effect of SRF in HCC cell growth and proliferation, and whether HCC cells expressing SRF were protected from apoptosis induced by chemotherapeutic agent, sorafenib.

\section{Materials and methods}

Patients and tissue specimens. This study was approved by the Institutional Review Board (IRB) of Chonbuk National University Hospital. A written informed consent was waived by the IRB due to the retrospective nature of the current study. A total of 146 cases of HCC patients, who had been diagnosed between January, 1999 and November, 2009 and for whom diagnostic HE stained slides and paraffin-embedded tissue blocks were available, were included in this study. In each case, clinicopathological features including age at diagnosis, gender, etiology (viral vs. non-viral), preoperative serum alpha-fetoprotein (AFP) and albumin levels, background liver disease, Edmonson-Steiner grade, and presence of microvessel invasion, intrahepatic metastasis and ascites were obtained from a review of medical records. All of these cases were reclassified according to the World Health Organization (WHO) classification, and tumors were staged according to the 2010 American Joint Committee on Cancer tumor-node-metastasis classification (24).

Cell culture, stable transfection and drug information. The human HCC cell line HLE, was purchased from the Health Science Research Resources Bank (Osaka, Japan). In addition, we used the sarcomatoid HCC cell line, designated as SH-J1, which was established in our laboratory (25). The HCC cells were grown in Dulbecco's modified Eagle's medium (DMEM) supplemented with penicillin and streptomycin $(100 \mathrm{U} / \mathrm{ml})$ and $10 \%$ fetal bovine serum (Gibco BRL, Gaithersburg, MD) at $37^{\circ} \mathrm{C}$ in a humidified $5 \% \mathrm{CO}_{2}$ incubator. A previously described method was used in performance of stable transfection of sense SRF and antisense SRF (18). We stably transfected epithelial HLE cell with SRF cDNA. Alternatively, we stably transfected sarcomatoid SH-J1 cells with SRF antisense cDNA. The anticancer drug, sorafenib was purchased from Santa Cruz Biotechnology (Santa Cruz Biotechnology, Santa Cruz, CA).

Immunohistochemistry and scoring. Immunohistochemical staining was performed with $3.0 \mathrm{~mm}$ tumor cores for tissue microarray (26) by a polymer intense detection system using the Bond-Max Automatic stainer (Leica Microsystems Inc., Bannockburn, IL) in accordance with the manufacturer's instructions. Following antigen retrieval in a microwave oven for $12 \mathrm{~min}$ in $0.01 \mathrm{~mol}$ citrate buffer ( $\mathrm{pH} \mathrm{6.0)}$, cells were incubated with anti-SRF (Santa Cruz Biotechnology), anti-Snail (Abcam, Cambridge, UK) antibody for $20 \mathrm{~min}$. The samples that were subjected to immunostaining were rated according to a score calculated by multiplying the area score by the intensity score of the staining. The area of staining was scored as follows: 0 (<10\% of the cancer cells), 1 (10-29\%), 2 (30-59\%) or $3(\geq 60 \%)$. The intensity of the cell nuclear staining was grouped into four categories: 0 , none; 1 , weak; 2 , moderate; 3 , strong. If the score was $\geq 2$, the tumor was considered positive; otherwise, the tumor was considered negative.

Western blot analysis. Protein extraction was performed with PRO-PREP Protein Extraction Solution (iNtRON Biotechnology Inc., Sungnam, Korea). Membranes were incubated with anti-SRF, anti-vimentin (Santa Cruz Biotechnology), anti-Snail (Abcam), anti-E-cadherin and anti-N-cadherin (BD Biosciences, San Jose, CA) antibodies overnight at $4^{\circ} \mathrm{C}$. Subsequently, membranes were incubated for $1 \mathrm{~h}$ at room temperature with corresponding secondary antibodies, and the immune complexes were visualized using the ECL detection system (Amersham Biosciences, Buckinghamshire, UK) and exposed to a luminescent image analyzer (LAS-3000, Fuji Film, Tokyo, Japan) as described previously (18).

Cell proliferation assay. The effects of the SRF gene on cell proliferation were assessed by MTT (3-(4,5-dimethylthiazol2-yl)-2,5-diphenyltetrazolium bromide) (Sigma, St. Louis, MO) and colony survival assay as described previously $(18,27)$. The HCC cells ( 3 or $5 \times 10^{3} /$ well) were grown on a 96 -well culture plate for $96 \mathrm{~h}$. MTT was added to each well. The plates were then incubated at $37^{\circ} \mathrm{C}$ for $2 \mathrm{~h}$. MTT was measured colorimetrically using a microtiter plate reader (Bio-Rad, Richmond, CA) at $560 \mathrm{~nm}$. Cytotoxicity studies were performed using MTT readings to represent the percentage of growth inhibited by increasing drug concentrations. HCC cells were incubated with sorafenib for 0.5-3 days in 96-well plates. Cytotoxicity was evaluated by comparing inhibitory concentration $50 \%$ $\left(\mathrm{IC}_{50}\right)$ values. The colony survival assay was performed in 12-well culture plates and cells were treated with sorafenib $24 \mathrm{~h}$ after cell seeding. Media and drugs were replaced every $72 \mathrm{~h}$ and colonies were stained after 10 days.

Cell cycle analysis. HCC cells were incubated with 5 or $10 \mu \mathrm{M}$ of sorafenib for $24 \mathrm{~h}$. Cells were collected and washed with PBS, fixed in $70 \%$ ethanol, treated with DNase-free RNase, and stained with $10 \mu \mathrm{g} / \mathrm{ml}$ propidium iodide (Sigma) at $37^{\circ} \mathrm{C}$ for $30 \mathrm{~min}$. The cell cycle phase distribution was determined by a FACStar flow cytometer (Becton-Dickinson, San Jose, CA) and analyzed using Lysis II and CellFIT software (Becton-Dickinson) or ModFit software (Verity Software House Inc., Topsham, ME).

Statistical analysis. Values are expressed as mean \pm SD. Statistical significance was determined by $\chi^{2}$ test and appropriate Student's t-test. P-values less than 0.05 were considered to be statistically significant. All experiments were repeated a minimum of three times.

\section{Results}

SRF was upregulated in HCC tissues. First, we evaluated the expression of SRF and Snail, which are well-known inducers of EMT, by immunohistochemistry in 146 pairs of HCC tissue and corresponding non-tumorous liver tissue 

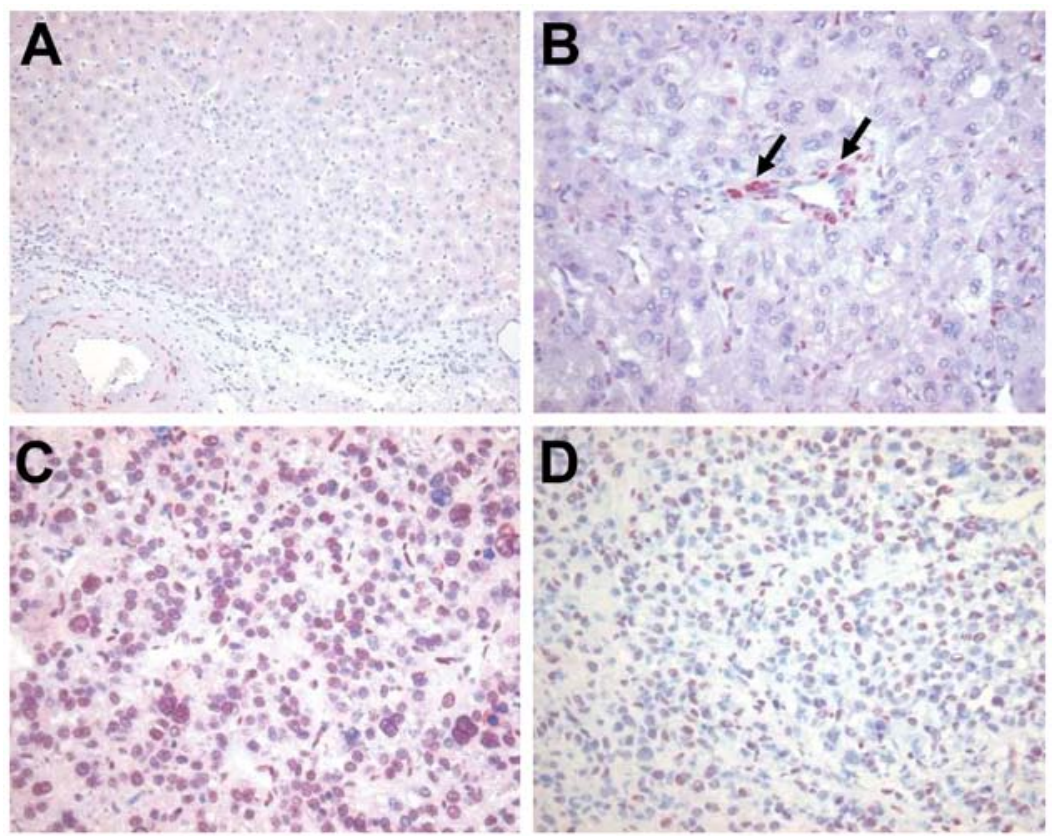

Figure 1. Immunohistochemical expression of SRF in hepatocellular carcinoma (HCC). (A) Normal hepatocytes showed no immunoreactivity for SRF. (B) Negative expression of SRF in low grade HCC. Note the strong nuclear expression of SRF in the smooth muscle cells of a blood vessel (arrows) and endothelial cells. (C) The nuclei of tumor cells showed a strong immunoreactivity for SRF in high grade HCC. (D) Snail expression in high grade HCC.

A

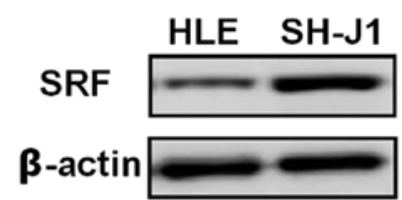

B

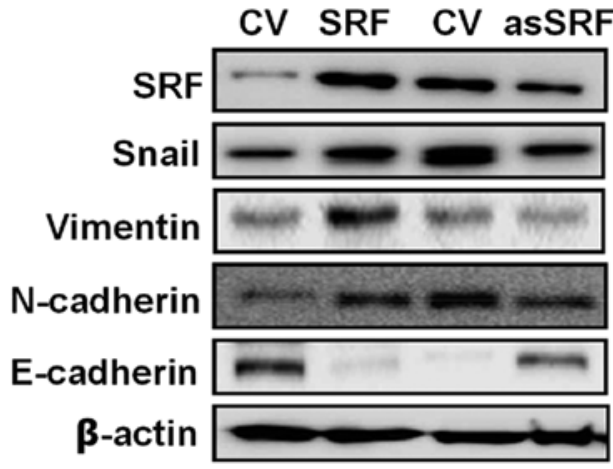

Figure 2. (A) The expression level of the SRF protein was higher in the sarcomatoid SH-J1 cell line than that in the HLE cell line. (B) Stable expression of SRF in HLE cells induced a reduction in E-cadherin expression and an increase in the expression of mesenchymal markers. Conversely, the inhibition of SRF expression in SH-J1 cells decreased the expression of mesenchymal markers. Furthermore, inhibition of SRF in SH-J1 cells restored the expression of E-cadherin.

from the same patient. Expression levels of SRF and Snail were detected in $37.6 \%$ ( 55 of 146 cases) and in $12.3 \%$ (18 of 146 cases) of the HCCs, respectively. None of the non-tumor liver tissues exhibited any SRF or Snail expression (Fig. 1). In addition to the tumor cells, SRF expression was observed in the smooth muscle cells of blood vessels and in stromal cells as described previously (17). To elucidate the significance of SRF expression in HCCs, a correlation between SRF and the major clinicopathological features was evaluated (Table I). The clinicopathological analysis revealed that SRF expression in HCC was significantly associated with high histological grade $(\mathrm{p}=0.015)$. SRF expression also closely correlated with the expression of Snail $(\mathrm{p}<0.001)$. However, SRF expression was not significantly correlated with other clinicopathological parameters such as age, gender, etiology, intrahepatic metas- tasis, microvessel invasion, presence of ascites or preoperative albumin and AFP level.

Overexpression of SRF-induced EMT in HCC cells. Because SRF expression was significantly associated with tumor de-differentiation and Snail expression, we examined the effect of SRF on EMT in HCC cells. We evaluated the expression of SRF in human HCC cell lines using western blot analysis. The expression level of the SRF protein was higher in the sarcomatoid SH-J1 cell line, which already exhibits mesenchymal-like cell features, than that in the HLE cell line (Fig. 2A). Stable expression of SRF in HLE cells induced a loss of endogenous E-cadherin expression, a representative epithelial adhesion molecule, and an increase in the expression of mesenchymal markers (vimentin, N-cadherin) concomitantly with the 
Table I. Clinicopathologic characteristics and correlation with SRF and Snail expression in HCC.

\begin{tabular}{|c|c|c|c|c|c|}
\hline \multirow[b]{2}{*}{ Characteristics } & \multirow[b]{2}{*}{ No. } & \multicolumn{2}{|c|}{ SRF } & \multicolumn{2}{|c|}{ Snail } \\
\hline & & Positive & P-value & Positive & P-value \\
\hline \multicolumn{6}{|l|}{ Age (years) } \\
\hline$<55$ & 58 & 26 & 0.147 & 9 & 0.341 \\
\hline$\geq 55$ & 88 & 29 & & 9 & \\
\hline \multicolumn{6}{|l|}{ Gender } \\
\hline Male & 124 & 47 & 0.891 & 15 & 0.840 \\
\hline Female & 22 & 8 & & 3 & \\
\hline \multicolumn{6}{|l|}{ Etiology } \\
\hline Non-viral & 30 & 9 & 0.331 & 13 & 0.417 \\
\hline Viral & 116 & 46 & & 5 & \\
\hline \multicolumn{6}{|l|}{$\begin{array}{l}\text { Preoperative } \\
\text { albumin }(\mathrm{g} / \mathrm{dl})\end{array}$} \\
\hline$\geq 3.5$ & 130 & 52 & 0.098 & 18 & 0.112 \\
\hline$<3.5$ & 16 & 3 & & 0 & \\
\hline \multicolumn{6}{|l|}{ Preoperative } \\
\hline \multicolumn{6}{|l|}{$\operatorname{AFP}(\mathrm{ng} / \mathrm{ml})$} \\
\hline$\leq 100$ & 96 & 35 & 0.675 & 11 & 0.658 \\
\hline$>100$ & 50 & 20 & & 7 & \\
\hline \multicolumn{6}{|l|}{ Liver cirrhosis } \\
\hline Absence & 76 & 32 & 0.249 & 12 & 0.185 \\
\hline Presence & 70 & 23 & & 6 & \\
\hline \multicolumn{6}{|l|}{ Ascites } \\
\hline Absence & 137 & 53 & 0.323 & 17 & 0.909 \\
\hline Presence & 9 & 2 & & 1 & \\
\hline \multicolumn{6}{|l|}{$\begin{array}{l}\text { Microvessel } \\
\text { invasion }\end{array}$} \\
\hline Absence & 52 & 20 & 0.883 & 3 & 0.073 \\
\hline Presence & 94 & 35 & & 15 & \\
\hline \multicolumn{6}{|l|}{ Histologic grade } \\
\hline 1 and 2 & 95 & 29 & 0.015 & 6 & 0.003 \\
\hline 3 and 4 & 51 & 26 & & 12 & \\
\hline \multicolumn{6}{|l|}{ pT stage } \\
\hline $\mathrm{pT} 1$ & 46 & 18 & 0.943 & 3 & 0.320 \\
\hline pT2 & 69 & 25 & & 11 & \\
\hline pT3 and 4 & 31 & 12 & & 4 & \\
\hline \multicolumn{6}{|l|}{$\begin{array}{l}\text { Intrahepatic } \\
\text { metastasis }\end{array}$} \\
\hline Absence & 95 & 36 & 0.939 & 13 & 0.497 \\
\hline Presence & 51 & 19 & & 5 & \\
\hline \multicolumn{6}{|l|}{ Snail expression } \\
\hline Negative & 128 & 41 & $<0.001$ & & \\
\hline Positive & 18 & 14 & & & \\
\hline
\end{tabular}

increased expression of Snail. Conversely, the inhibition of SRF expression in the SH-J1 cells by antisense SRF cDNA decreased the expression of mesenchymal markers and Snail. Furthermore, inhibition of SRF in SH-J1 cell lines lacking E-cadherin protein restored the expression of E-cadherin (Fig. 2B). These results suggested that SRF plays an important role in the EMT process in HCC.
Overexpression of SRF increased cell growth and proliferation. SRF-overexpressing HLE cells showed significantly increased cell proliferation and colony forming capacity when compared to those of the control vector cells $(\mathrm{p}<0.05$; Fig. 3A and C). In contrast, both the cell proliferation and colony-forming capacity of the sarcomatoid SH-J1 cells were decreased when expression of SRF was blocked by antisense SRF cDNA ( $<0.05$; Fig. 3B and D).

SRF conferred sorafenib resistance to HCC cells. To investigate the response of HCC cells to genotoxic stress, sorafenib was used to induce cell death. After sorafenib exposure, viability of HLE and SH-J1 cells decreased in a time- and dose-dependent manner as measured by the MTT test (Fig. 4). The level of sorafenib-induced cell death was lower in SH-J1 cells when compared to that of HLE cells (Fig. 4) suggesting that higher SRF-expressing, mesenchymal-like SH-J1 cells may be less responsive to sorafenib-induced cell death. Forced overexpression of SRF in HLE cells prevented sorafenib-induced cell death (Fig. 5A and C). Conversely, SRF depletion in SH-J1 cells substantially enhanced the apoptotic effect of sorafenib (Fig. 5B and D). The reduction in cell number observed in HLE and $\mathrm{SH}-\mathrm{J} 1$ cells prompted us to evaluate whether this effect was due to increased cell death, decreased cell proliferation or both. FACS analysis of HCC cells treated with sorafenib showed a distinct, quantifiable population of cells with DNA contents below the G1-phase level (a sub-G1), indicating increased cell death by apoptosis (Fig. 6). SRF-overexpressing HLE cells showed lower accumulation of HCC cells in the Sub G1 phase when compared with that of controls during sorafenib treatment. On the contrary, SRF depletion in SH-J1 cells increased cells in Sub G1 phase when compared with that of controls. Altogether, these results suggest that the cytotoxic effect of sorafenib in HCC is correlated with the expression level of SRF.

\section{Discussion}

SRF is a member of the MADS-box family of transcription factors and is one of the most studied DNA-binding proteins in the human proteome (11-15). Although its roles in murine embryogenesis and the expression of smooth muscle marker genes have been studied and are increasingly understood, the relevance of SRF in human cancer is largely unknown. Here, we provide evidence that aberrant expression of SRF in HCC protects against apoptosis induced by sorafenib therapy. Our data demonstrate the following: i) expression of SRF was significantly elevated in HCC tissues and associated with high grade HCC; ii) SRF expression was closely correlated with the expression of Snail, a transcriptional factor known to suppress E-cadherin, a protein involved in EMT (28); iii) overexpression of SRF in HLE cells led to EMT, as well as increased cell growth; and iv) overexpression of SRF also led to significantly reduced cytotoxic effects of sorafenib in HLE cells. Furthermore, depletion of endogenous SRF by the SRF antisense cDNA in the sarcomatoid SH-J1 cells led to enhanced cytotoxic effects of sorafenib accompanied by a decrease in the expression of mesenchymal markers. These findings strongly suggest that expression of SRF in HCC cells with an epithelial phenotype not only promotes phenotypic transition to a less differentiated, mesenchymal pattern, but 


\section{HLE}
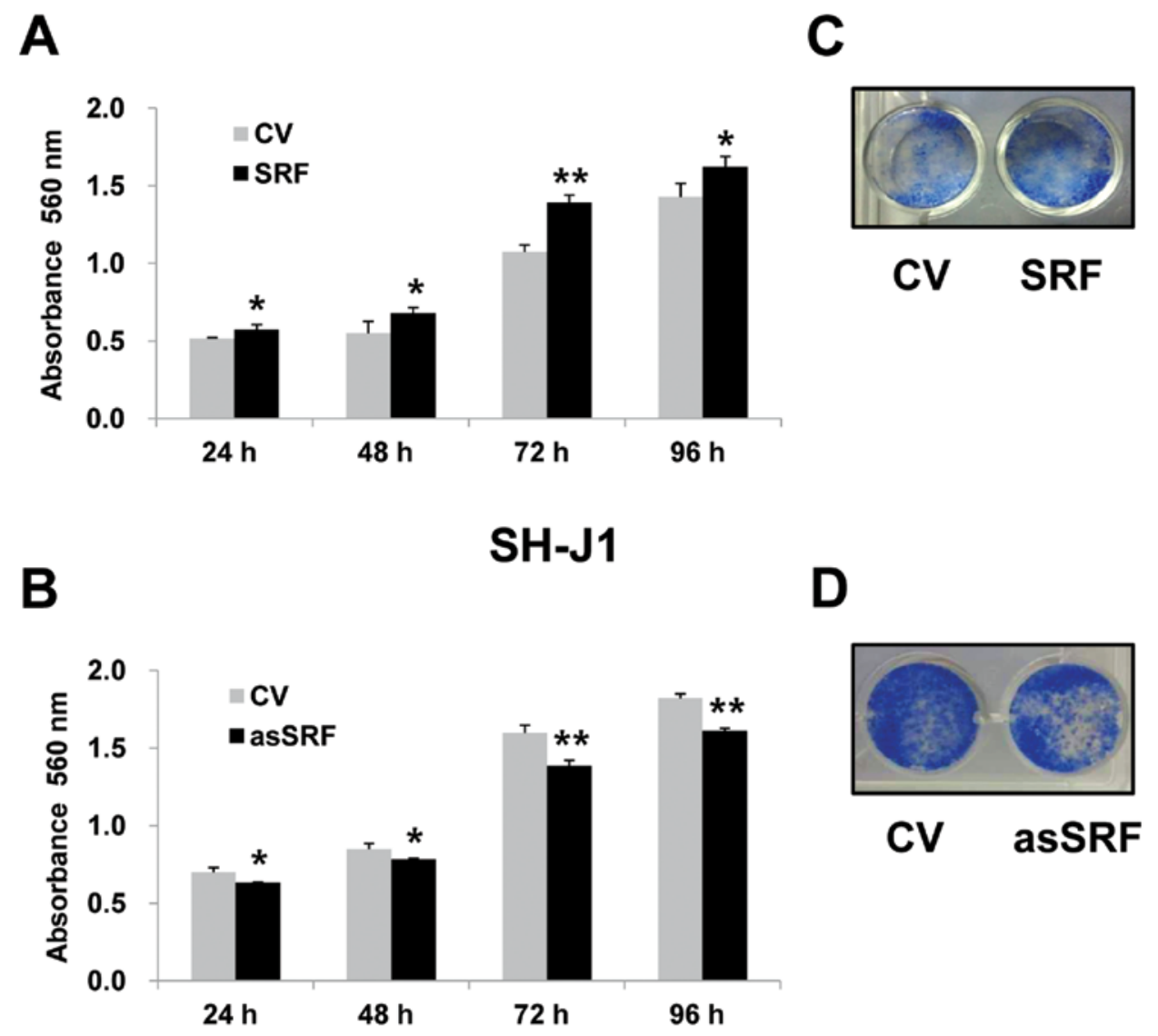

Figure 3. (A and C) Forced expression of SRF significantly enhanced the cell proliferation and colony-forming capacity of HLE cells $\left({ }^{*} \mathrm{p}<0.05\right.$, ${ }^{* *} \mathrm{p}<0.005$ ). (B and D) Conversely, the cell proliferation and colony-forming capacity of SH-J1 cells were decreased when SRF expression was inhibited $\left({ }^{*} \mathrm{p}<0.05,{ }^{* *} \mathrm{p}<0.005\right)$.
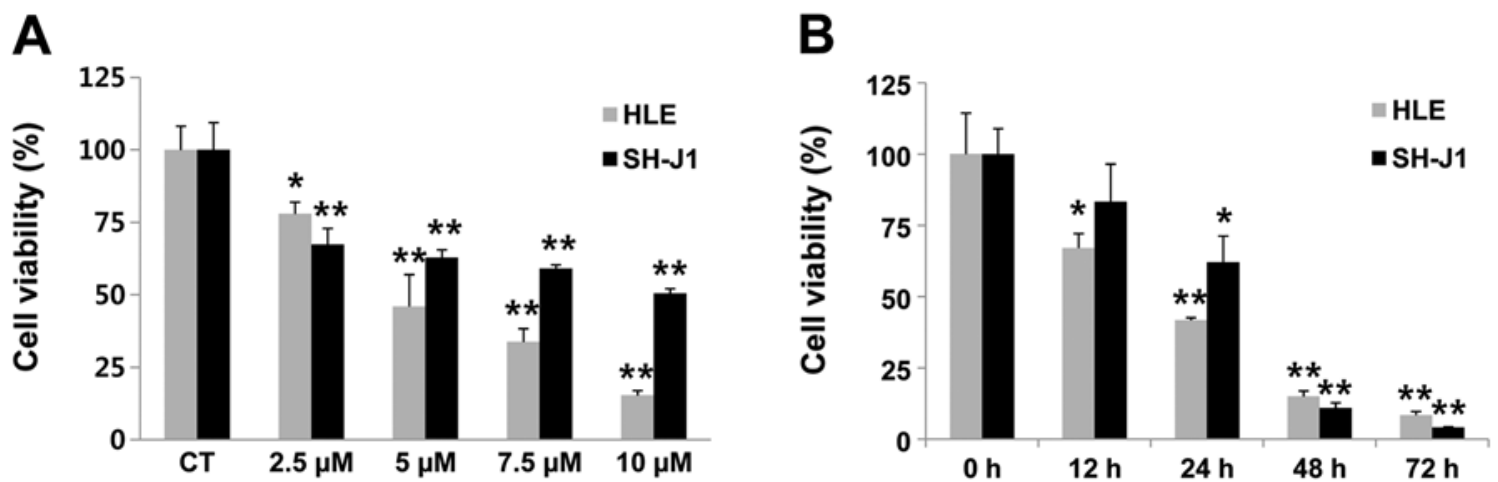

Figure 4. (A) Dose and (B) time-dependent inhibitory effects of sorafenib on cell growth in HCC cell lines, as measured by MTT assay ("p $\left.<0.05,{ }^{* *} \mathrm{p}<0.005\right)$.

also provides protection against apoptotic cell death induced by sorafenib therapy. Modulation of SRF expression can affect the sensitivity of HCC cells to sorafenib, which suggests that a reduction of SRF in combination with sorafenib could be a potential therapeutic approach in the treatment of HCC.

In human cancer, aberrant expression of SRF has been implicated in carcinogenesis and tumor progression (16-20,29). We have previously shown, by western blot analysis of protein isolated from frozen HCC samples, that SRF is specifically upregulated in tumor tissues (30). In this study, we found that SRF expression correlates with the de-differentiation of cancer cells and is associated with the expression of Snail in surgically-resected human HCC samples. We also found that forced expression of SRF in HLE cells induces the loss of E-cadherin and the increased expression of mesenchymal markers. In agreement with our findings, SRF has been known to induce the mesenchymal transition of epithelial tumor cells in a multistage model of mouse skin carcinogenesis (16). We have previously 


\section{HLE}

A

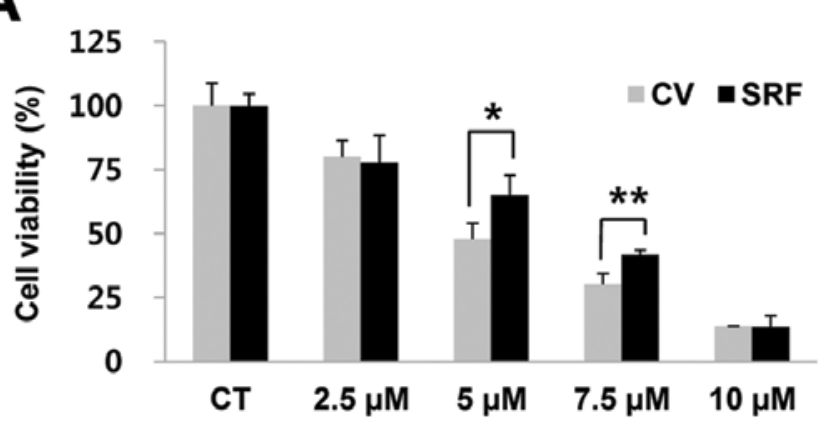

SH-J1

B

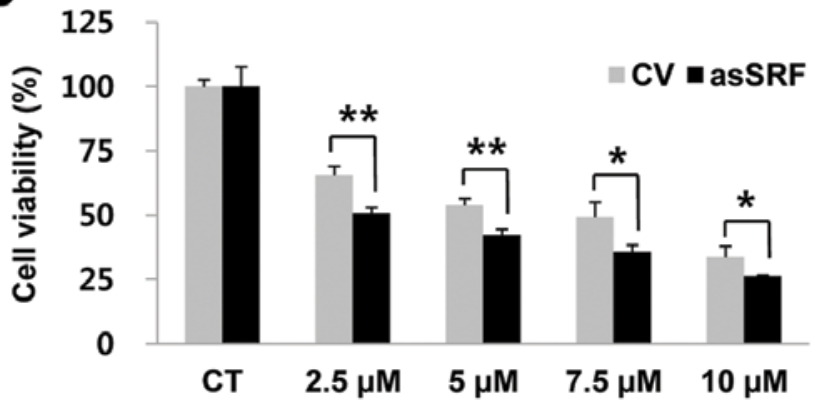

C

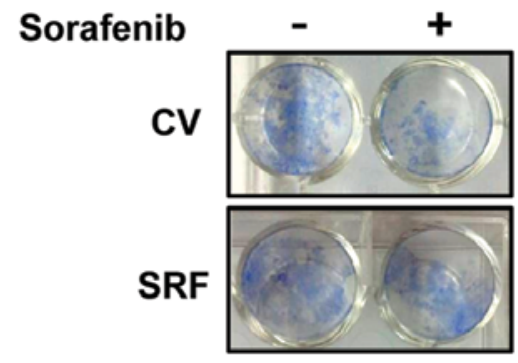

D

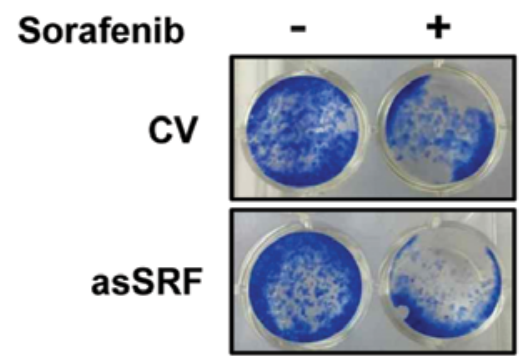

Figure 5. Expression of SRF modulated the sensitivity of HCC cells to sorafenib. (A and C) Forced expression of SRF in HLE cells significantly blocked the inhibitory effect of the cell proliferation and colony-forming capabilities by sorafenib when compared with those of the control vector $(\mathrm{CV})\left({ }^{*} \mathrm{p}<0.05\right.$, $\left.^{*} \mathrm{p}<0.005\right)$. (B and D). Conversely, SRF depletion in SH-J1 cells significantly enhanced the inhibitory effect of the cell proliferation and colony forming capabilities by sorafenib when compared to those of the CV $\left({ }^{*} \mathrm{p}<0.05,{ }^{* *} \mathrm{p}<0.005\right)$.

demonstrated that overexpression of SRF in HCC cells leads to changes in cell morphology, increased actin polymerization and enhanced cell migration and invasiveness; all are indicators of EMT (18). We have also shown that expression of SRF in metastatic colorectal carcinoma cells is significantly higher than that of primary tumors and is associated with modulation of E-cadherin/ $\beta$-catenin expression in colorectal carcinoma cells (19). In addition, overexpression of SRF increased tumor cell migration and invasion through upregulation of MMP-2 and -9 in HCC cells (30). Collectively, our findings indicate that SRF plays a crucial role in HCC progression, specifically, the mesenchymal transition of epithelial tumor cells.

Sorafenib is an orally active multikinase inhibitor, approved for the treatment of unresectable HCC, and has been demonstrated to provide a survival advantage for HCC patients in a randomized trial $(3,23)$. However, its clinical benefits remain modest due to the acquired resistance developed by many patients (31). Emerging evidence has revealed that EMT of carcinoma cells may be an important source of resistance to various types of anticancer drugs (7,32-34), including sorafenib (31). Chemoresistance to paclitaxel induces EMT and enhances metastatic potential for epithelial ovarian carcinoma cells and chronic exposure of oxaliplatin induces EMT with chemoresistance in colorectal cancer cell lines $(32,33)$. Moreover, EMT is observed in gefitinib-resistant lung cancer cells with a decrease in expression of E-cadherin and an increase in expres- sion of mesenchymal markers (34). These studies strongly demonstrate that EMT is correlated with chemoresistance. The close association between EMT and sorafenib resistance, either as a trigger or as a result, has been reported (31,35-37). Sorafenib-resistant HCC cells demonstrate altered morphological features similar to characteristics associated with EMT, such as loss of E-cadherin and high expression of vimentin (35). $\alpha \mathrm{B}$-crystallin induced EMT and resistance to sorafenib through activation of the extracellular-regulated protein kinase cascade in HCC (36). Nagai et al demonstrated that sorafenib exerts a potent inhibitory effect against EMT by inhibiting MAPK signaling and Snail expression in HCC (37). We found that expression of SRF was significantly associated with high grade HCC. Consistent with our findings, expression of SRF was significantly associated with high Gleason histologic score in prostate cancer $(38,39)$. We also found that sarcomatoid SH-J1 cells showed higher expression of SRF than epithelial HLE cells and SH-J1 cells were less affected by sorafenib treatment than HLE cells. Modulation of SRF expression in HLE and SH-J1 cells also affected the apoptotic effect of sorafenib, as demonstrated by MTT and colony-forming assays. These findings indicate that the cytotoxic effect of sorafenib depends on the expression level of SRF. Together with the fact that carcinomas showing EMT are mostly high grade carcinomas, our results indicate that SRF-expressing, high grade HCC cells are more resistant to sorafenib therapy, which is in accordance with 

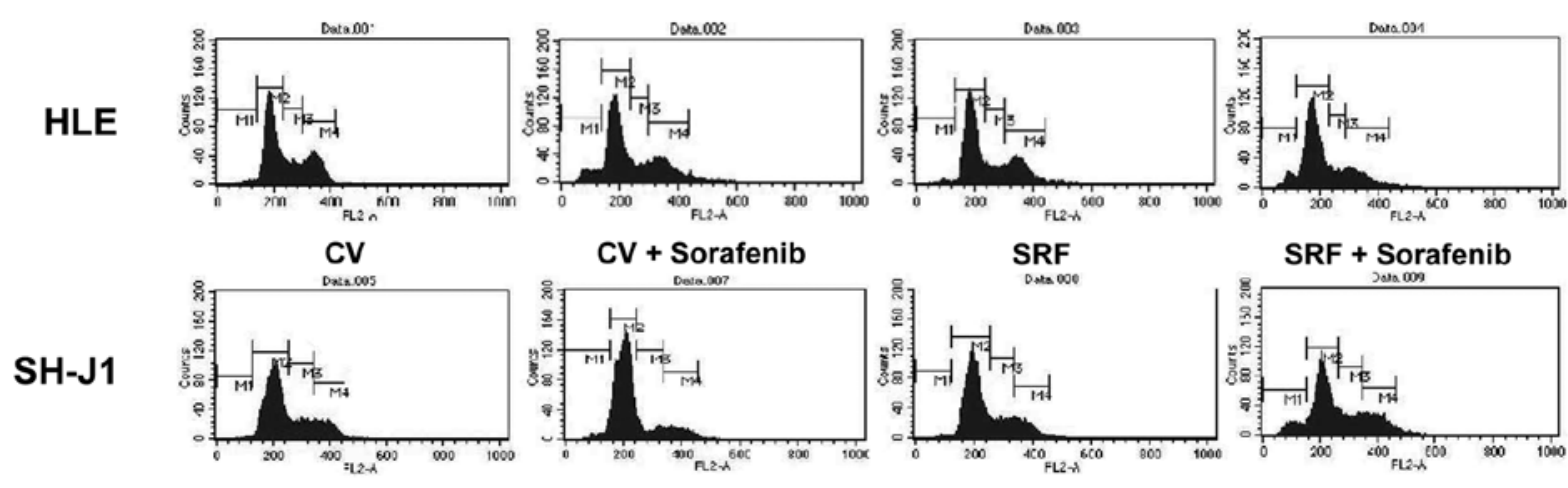

CV

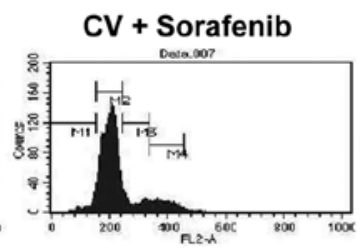

CV + Sorafenib

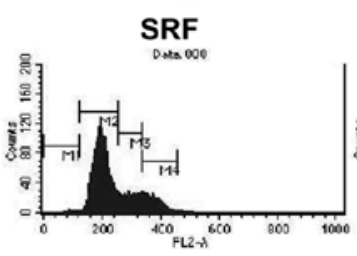

asSRF

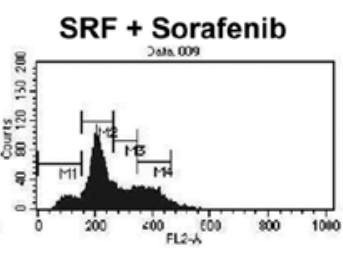

asSRF + Sorafenib
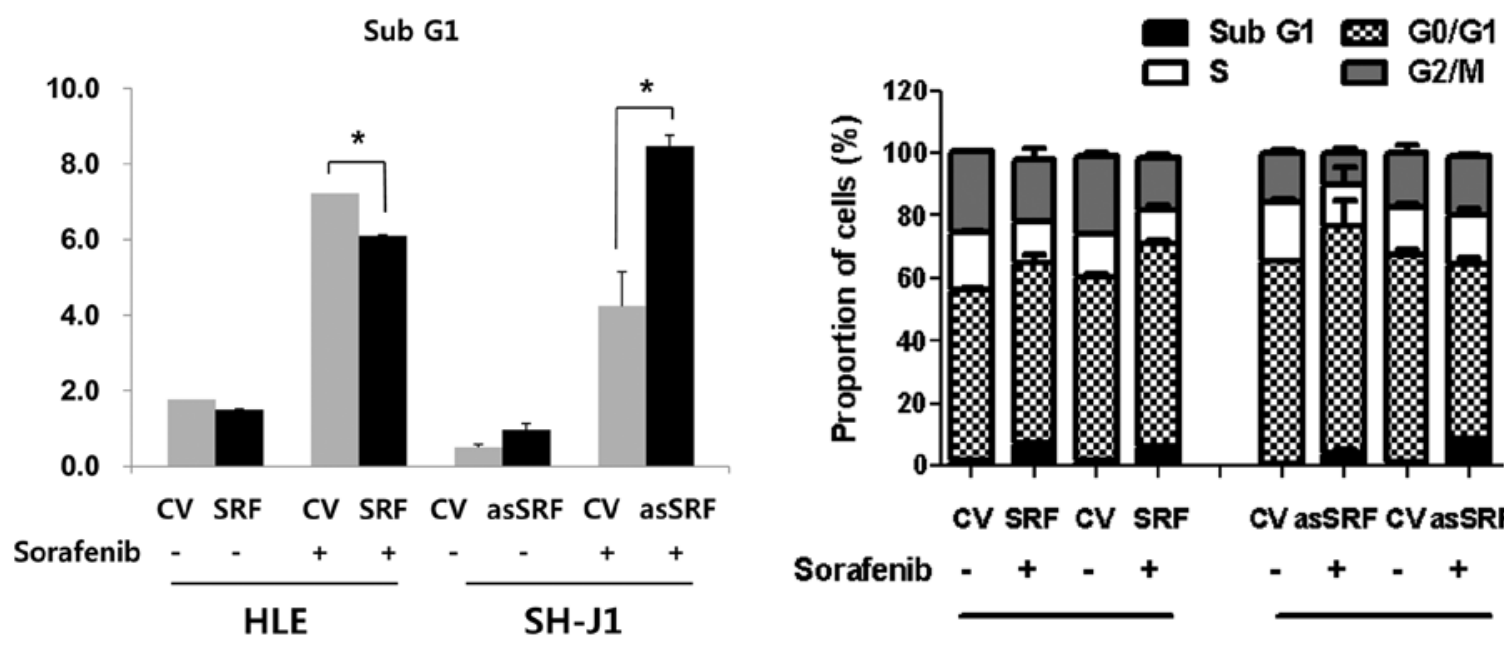

HLE

SHJ1

Figure 6. SRF-overexpressing HLE cells showed lower accumulation of HCC cells in Sub G1 phase when compared with that of controls during the sorafenib treatment. Conversely, SRF depletion in SH-J1 cells increased the proportion of cells in Sub G1 phase when compared with that of controls ( $\mathrm{p}<<0.05)$.

clinical observations that high grade HCC is more refractory to chemotherapy (40), and that the development of insensitivity to chemotherapy was a result of EMT induction (7,32-36). Therefore, our results support the notion that the EMT caused by SRF may initiate the development of chemoresistance to sorafenib in HCC.

The present study demonstrated that the cell proliferation and colony-forming capacity of sarcomatoid SH-J1 cells were decreased when the expression of SRF was blocked by antisense SRF cDNA. This finding is in agreement with that of a previous study demonstrating that SRF inhibition by siRNA or small-molecule inhibitor CCG-1423 induces decreased cellular proliferation in prostate cancer cells (40). Similarly, RNA interference targeting SRF caused a reduction of S-phase cells, a decrease in cell number and reduced vitality of HCC cells (20). We found that the downregulation of SRF in SH-J1 cells led to partial reversal of the EMT phenotype, resulting in decreased expression of mesenchymal markers and restored expression of the E-cadherin. Moreover, SRF depletion in SH-J1 cells substantially enhanced the apoptotic effect of sorafenib. We have also shown that inhibition of SRF expression resulted in significantly decreased migration and invasion of HCC cells (18). Similar to our findings, depletion of myocardin-related transcription factors or SRF via RNA interference has been demonstrated to reduce the metastatic potential of cancer cells by blocking target genes important in cell adhesion, spreading, invasion and motility (41). The miR-122 microRNA that targets SRF degradation is downregulated in HCC tissues compared to non-tumorous liver tissue, whereas SRF is predominantly expressed in HCC tissues (42). The growth and clonogenic survival of miR-122 expressing HCC cells were significantly reduced upon treatment with sorafenib. Additionally, ectopic expression of SRF reversed the inhibitory effect of miR-122 on HCC cell growth and clonogenic survival (42). Recently, Yu et al demonstrated that increased activity of SRF and transcription of multiple genes caused by FGFR-4 $\mathrm{Arg}^{388}$ are correlated with aggressive clinical behavior in prostate cancer patients. Furthermore, knockdown of FGFR-4 $\mathrm{Arg}^{388}$ in prostate cancer cells decreases proliferation and invasion in vitro and primary tumor growth and metastasis in vivo (38). These reports and our results provide a rationale for novel therapeutic approaches of SRF targeting for HCC.

In conclusion, we have shown for the first time that SRF expression has a distinct impact on drug resistance to a chemotherapeutic agent, sorafenib, in HCC cell lines. Our results also suggest that the degree of cytotoxicity associated with sorafenib depends not only on the expression of SRF, but, more precisely, on the occurrence of EMT caused by SRF in HCC cells. 


\section{Acknowledgements}

This study was supported by the Fund of the Research Institute of Clinical Medicine, Chonbuk National University Hospital and by the National Research Foundation of Korea Grant funded by the Korean Government (no. 2012-0009320). Tissue samples were provided by the Chonbuk National University Hospital, a member of the National Biobank of Korea, which is supported by the Ministry of Health, Welfare and Family Affairs.

\section{References}

1. El-Serag HB: Epidemiology of viral hepatitis and hepatocellular carcinoma. Gastroenterology 142: 1264-1273, 2012.

2. Ferlay J, Shin HR, Bray F, Forman D, Mathers C and Parkin DM: Estimates of worldwide burden of cancer in 2008 GLOBOCAN 2008. Int J Cancer 127: 2893-2917, 2010.

3. Avila MA, Berasain C, Sangro B and Prieto J: New therapies for hepatocellular carcinoma. Oncogene 25: 3866-3884, 2006.

4. Polyak K and Weinberg RA: Transitions between epithelial and mesenchymal states: acquisition of malignant and stem cell traits. Nat Rev Cancer 9: 265-273, 2009.

5. Kalluri R and Weinberg RA: The basics of epithelial-mesenchymal transition. J Clin Invest 119: 1420-1428, 2009.

6. Creighton CJ, Li X, Landis M, et al: Residual breast cancers after conventional therapy display mesenchymal as well as tumor-initiating features. Proc Natl Acad Sci USA 106 $13820-13825,2009$.

7. Singh A and Settleman J: EMT, cancer stem cells and drug resistance: an emerging axis of evil in the war on cancer. Oncogene 29: 4741-4751, 2010.

8. Kurrey NK, Jalgaonkar SP, Joglekar AV, et al: Snail and slug mediate radioresistance and chemoresistance by antagonizing P53-mediated apoptosis and acquiring a stem-like phenotype in ovarian cancer cells. Stem Cells 27: 2059-2068, 2009.

9. Wang Z, Li Y, Kong D, et al: Acquisition of epithelialmesenchymal transition phenotype of gemcitabine-resistant pancreatic cancer cells is linked with activation of the notch signaling pathway. Cancer Res 69: 2400-2407, 2009.

10. Peinado H, Olmeda D and Cano A: Snail, ZEB and bHLH factors in tumour progression: an alliance against the epithelial phenotype? Nat Rev Cancer 7: 415-428, 2007.

11. Treisman R: Identification of a protein-binding site that mediates transcriptional response of the c-fos gene to serum factors. Cell 46: 567-574, 1986.

12. Treisman R: Identification and purification of a polypeptide that binds to the c-fos serum response element. EMBO $\mathrm{J} 6$ : 2711-2717, 1987

13. Shore P and Sharrocks AD: The MADS-box family of transcription factors. Eur J Biochem 229: 1-3, 1995.

14. Modak C and Chai J: Serum response factor: look into the gut. World J Gastroenterol 16: 2195-2201, 2010.

15. Miano JM: Role of serum response factor in the pathogenesis of disease. Lab Invest 90: 1274-1284, 2010.

16. Psichari E, Balmain A, Plows D, Zoumpourlis V and Pintzas A: High activity of serum response factor in the mesenchymal transition of epithelial tumor cells is regulated by RhoA signaling. J Biol Chem 277: 29490-29495, 2002.

17. Park MY, Kim KR, Park HS, et al: Expression of the serum response factor in hepatocellular carcinoma: Implications for epithelial-mesenchymal transition. Int J Oncol 31: 1309-1315, 2007.

18. Kwon CY, Kim KR, Choi HN, et al: The role of serum response factor in hepatocellular carcinoma: Implications for disease progression. Int J Oncol 37: 837-844, 2010.

19. Choi HN, Kim KR, Lee JH, et al: Serum response factor enhances liver metastasis of colorectal carcinoma via alteration of the E-cadherin/ $\beta$-catenin complex. Oncol Rep 21: 57-63, 2009.

20. Farra R, Dapas B, Pozzato G, et al: Serum response factor depletion affects the proliferation of the hepatocellular carcinoma cells HepG2 and JHH6. Biochimie 92: 455-463, 2010.

21. Wilhelm S, Carter C, Lynch M, et al: Discovery and development of sorafenib: a multikinase inhibitor for treating cancer. Nat Rev Drug Discov 5: 835-844, 2006.
22. Wilhelm SM, Adnane L, Newell P, Villanueva A, Llovet JM and Lynch M: Preclinical overview of sorafenib, a multikinase inhibitor that targets both Raf and VEGF and PDGF receptor tyrosine kinase signaling. Mol Cancer Ther 7: 3129-3140, 2008.

23. Kane RC, Farrell AT, Madabushi R, et al: Sorafenib for the treatment of unresectable hepatocellular carcinoma. Oncologist 14: $95-100,2009$

24. Edge SB, Byrd DR, Compton CC, Fritz AG, Greene FL and Trotti A: AJCC Cancer Staging Manual. 7th edition. Springer, New York, NY, 2010.

25. Kim DG, Park SY, Kim H, Chun YH, Moon WS and Park SH A comprehensive karyotypic analysis on a newly established sarcomatoid hepatocellular carcinoma cell line SH-J1 by comparative genomic hybridization and chromosome painting. Cancer Genet Cytogenet 132: 120-124, 2002.

26. Bae JS, Choi HN, Noh SJ, et al: Expression of K19 and K7 in dysplastic nodules and hepatocellular carcinoma. Oncol Lett 4: 213-220, 2012.

27. Kim KR, Choi HN, Lee HJ, et al: A peroxisome proliferatoractivated receptor $\gamma$ antagonist induces vimentin cleavage and inhibits invasion in high-grade hepatocellular carcinoma. Oncol Rep 18: 825-832, 2007.

28. Cano A, Pérez-Moreno MA, Rodrigo I, et al: The transcription factor Snail controls epithelial-mesenchymal transitions by repressing E-cadherin expression. Nat Cell Biol 2: 76-83, 2000.

29. Park SY, Jang KY, Kim YN, et al: Expression and prognostic significance of serum response factor in cholangiocarcinoma. Korean J Pathol 43: 517-522, 2009.

30. Kim KR, Bae JS, Choi HN, et al: The role of serum response factor in hepatocellular carcinoma: An association with matrix metalloproteinase. Oncol Rep 26: 1567-1572, 2011.

31. Gauthier A and Ho M: Role of sorafenib in the treatment of advanced hepatocellular carcinoma: an update. Hepatol Res 43: 147-154, 2013.

32. Kajiyama H, Shibata K, Terauchi M, et al: Chemoresistance to paclitaxel induces epithelial-mesenchymal transition and enhances metastatic potential for epithelial ovarian carcinoma cells. Int J Oncol 31: 277-283, 2007.

33. Yang AD, Fan F, Camp ER, et al: Chronic oxaliplatin resistance induces epithelial-to-mesenchymal transition in colorectal cancer cell lines. Clin Cancer Res 12: 4147-4153, 2006.

34. Rho JK, Choi YJ, Lee JK, et al: Epithelial to mesenchymal transition derived from repeated exposure to gefitinib determines the sensitivity to EGFR inhibitors in A549, a non-small cell lung cancer cell line. Lung Cancer 63: 219-226, 2009.

35. Van Malenstein H, Dekervel J, Verslype C, et al: Long-term exposure to sorafenib of liver cancer cells induces resistance with epithelial-to-mesenchymal transition, increased invasion and risk of rebound growth. Cancer Lett 329: 74-83, 2013.

36. Huang XY, Ke AW, Shi GM, et al: $\alpha \mathrm{B}$-crystallin complexes with $14-3-3 \zeta$ to induce epithelial-mesenchymal transition and resistance to sorafenib in hepatocellular carcinoma. Hepatology 57: 2235-2247, 2013

37. Nagai T, Arao T, Furuta K, et al: Sorafenib inhibits the hepatocyte growth factor-mediated epithelial mesenchymal transition in hepatocellular carcinoma. Mol Cancer Ther 10: 169-177, 2011.

38. Yu W, Feng S, Dakhova O, et al: FGFR-4 Arg ${ }^{388}$ enhances prostate cancer progression via extracellular signal-related kinase and serum response factor signaling. Clin Cancer Res 17: 4355-4366, 2011.

39. Prencipe M, Madden SF, O'Neill A, et al: Identification of transcription factors associated with castration-resistance: is the serum responsive factor a potential therapeutic target? Prostate 73: 743-753, 2013.

40. Pérez-Saborido B, de los Galanes SJ, Menéu-Díaz JC, et al: Tumor recurrence after liver transplantation for hepatocellular carcinoma: recurrence pathway and prognostic factors. Transplant Proc 39: 2304-2307, 2007.

41. Medjkane S, Perez-Sanchez C, Gaggioli C, Sahai E and Treisman R: Myocardin-related transcription factors and SRF are required for cytoskeletal dynamics and experimental metastasis. Nat Cell Biol 11: 257-268, 2009.

42. Bai S, Nasser MW, Wang B, et al: MicroRNA-122 inhibits tumorigenic properties of hepatocellular carcinoma cells and sensitizes these cells to sorafenib. J Biol Chem 284: 32015-32027, 2009. 